\title{
Reduction of Structural Vibrations by Passive and Semiactively Controlled Friction Dampers
}

\author{
L. Gaul and J. Becker \\ Institute of Applied and Experimental Mechanics, University of Stuttgart, Germany \\ Correspondence should be addressed to L. Gaul; gaul@iam.uni-stuttgart.de
}

Received 26 July 2013; Accepted 24 February 2014; Published 17 July 2014

Academic Editor: Nuno Maia

Copyright ( 2014 L. Gaul and J. Becker. This is an open access article distributed under the Creative Commons Attribution License, which permits unrestricted use, distribution, and reproduction in any medium, provided the original work is properly cited.

\begin{abstract}
Reduction of structural vibrations is of major interest in mechanical engineering for lowering sound emission of vibrating structures, improving accuracy of machines, and increasing structure durability. Besides optimization of the mechanical design or various types of passive damping treatments, active structural vibration control concepts are efficient means to reduce unwanted vibrations. In this contribution, two different semiactive control concepts for vibration reduction are proposed that adapt to the normal force of attached friction dampers. Thereby, semiactive control concepts generally possess the advantage over active control in that the closed loop is intrinsically stable and that less energy is required for the actuation than in active control. In the chosen experimental implementation, a piezoelectric stack actuator is used to apply adjustable normal forces between a structure and an attached friction damper. Simulation and experimental results of a benchmark structure with passive and semiactively controlled friction dampers are compared for stationary narrowband excitation. For simulations of the control performance, transient simulations must be employed to predict the achieved vibration damping. It is well known that transient simulation of systems with friction and normal contact requires excessive computational power due to the nonlinear constitutive laws and the high contact stiffnesses involved. However, commercial finite-element codes do not allow simulating feedback control in a general way. As a remedy, a special simulation framework is developed which allows efficiently modeling interfaces with friction and normal contact by appropriate constitutive laws which are implemented by contact elements in a finite-element model. Furthermore, special model reduction techniques using a substructuring approach are employed for faster simulation.
\end{abstract}

\section{Introduction}

Semiactive control strategies for vibration reduction offer interesting alternatives to passive means of damping enhancement or fully active vibration control (AVC). Hereby, the term semiactive means that passive system properties, such as friction, material damping, or fluid viscosity, are actively controlled. This intrinsically eliminates the problem of system destabilization due to spillover effects encountered in AVC applied to flexible structures $[1,2]$. Furthermore, semiactive control is more energy-efficient than fully active ones in general which is an important aspect from an application point of view. In exchange of these advantages, the achievable performance is limited by the effectiveness of the underlying passive damping mechanism. Though, they outperform passive vibration reduction means due to their ability to adapt to the instantaneous vibration state of the structure, this property links semiactive control concepts to the research area of smart/adaptive structures and adaptronics. Advantages over fully active control are that semiactive control is inherently fail-safe, guarantees stability, and introduces significant passive damping into the mechanical system, for example, by the attached friction damper in this contribution. Semiactive control concepts are probably most often applied to magneto- or electrorheological dampers, friction damping devices, or actively tuned absorbers with variable-stiffness dampers; see [3-11] for some examples. In this contribution, a semiactive control concept for a friction damper which is able to reduce structural vibrations of multiple modes is presented. The specific idea of using friction in joints for vibration damping by normal force control is reported first in [12], which subsequently inspired several researchers; see, for example, [13]. Two control algorithms for the semiactive vibration 
control of the normal force between a main structure and attached damper exploiting dry friction damping are investigated. The focus of this contribution is to damp several structural modes of beam.

\section{System Representation}

2.1. Beam Structure. A beam-like friction damper element is attached to a beam-like metal benchmark structure by a normal screw and an adaptive screw. The principle is depicted schematically in Figure 1; a picture of the experimental realization is shown in Figure 2. One screw is strongly tightened with a normal force of $F_{N, 2}=6000 \mathrm{~N}$ whereas the normal force $F_{N, 1}$ of the adaptive screw can be controlled by means of a piezoelectric ring stack actuator. For that purpose, the control measures the acceleration close to the tip.

2.2. Finite-Element Modeling. The structure is discretized by the finite-element (FE) method using ANSYS (Figure 3). The beam and the friction damper are modeled as independent substructures with solid elements having quadratic shape functions. The mesh size is chosen in accordance with Shannon's theorem. At the interface identical meshes are used to enable the application of a node-to-node contact formulation. After assembly the established system matrices in terms of mass and stiffness are transferred to MATLAB using the Structural Dynamics Toolbox. This procedure offers flexibility for applying different model reduction as well as applying control techniques. Reduced models with a minimal truncation error were found in a previous investigation and for more details it is referred to $[14,15]$ at this point.

In a generic way, the discretized structural dynamics of the two substructures, namely, the main structure and the attached friction damper beam, are given by

$$
\begin{aligned}
& {\left[\begin{array}{cc}
M^{(1)} & 0 \\
0 & M^{(2)}
\end{array}\right] \ddot{x}+\left[\begin{array}{cc}
K^{(1)} & 0 \\
0 & K^{(2)}
\end{array}\right] x+\left[\begin{array}{c}
B_{T}^{(1)} \\
B_{T}^{(2)}
\end{array}\right] F_{T}^{c}+\left[\begin{array}{c}
B_{N}^{(1)} \\
B_{N}^{(2)}
\end{array}\right] F_{N}^{c}} \\
& \quad=B\left[\begin{array}{l}
F_{N, 1} \\
F_{N, 2}
\end{array}\right]+F_{\text {exc }}
\end{aligned}
$$

where the nodal normal contact forces $F_{N}^{c}$ and tangential friction forces $F_{T}^{c}$ act as internal forces on the contact interface between main structure and damper beam. On the righthand side, the external forces appear, namely, the two pairs of clamping forces $F_{N, 1}, F_{N, 2}$ and dynamic excitation loads $F_{\text {exc }}$ (later, $F_{N, 1}$ is controlled).

Constitutive equations are implemented for the normal contact and the tangential contact in the interface by nodeto-node contact elements. The former is a bilinear stiffness relationship (Figure 4) that allows separation for a gap $g>g_{0}$ but penalizes penetration in two steps for $g<g_{0}$ and $g<g_{1}$. For the friction an elastoplastic model is used, which can be seen as two-dimensional version of a Jenkins element model depicted in Figure 4 for the one-dimensional case, that is, a series combination of a spring and a Coulomb element.
The nonlinear system of (1) is solved with the Newmark scheme and Newton equilibrium iterations at each fixed time step. Beforehand, substructure model reduction techniques are employed to reduce the dimensions of the system. For that purpose, an in-house simulation tool [14] is developed to facilitate general feedback control simulation, which is not supported by commercial FE codes. The model parameters including the contact parameters are updated by comparison of experimentally and numerically obtained FRFs (frequency response function) with impulse hammer excitation that cover a large range of constant clamping forces. An exemplary FRF is shown in Figure 5 where the nonlinear effects become visible by some unsymmetric peak forms (e.g., at $230 \mathrm{~Hz}$ ) and some higher harmonics peaks. From the FRF, the damping ratios are identified for the bending modes; see Figure 5. They show good agreement between simulation and experiment and significant damping is introduced by the friction damper if they are compared to the modal damping ratios of less than $0.15 \%$ found without attached damper.

\section{Semiactive Vibration Control}

Two controllers each consisting of an appropriate nonlinear control law plus an observer to estimate nonmeasurable variables required by the control are introduced in the following. The first control is denoted by hysteresis-optimal control and is motivated by experimental investigations. They show that relatively simple dynamical friction models are often capable of modeling the most dominant friction effects in structures with local joints [16]. Among others, the Jenkins element, as depicted in Figure 4, has proven its usability for that purpose and serves as base for the control derivation.

3.1. Hysteresis-Optimal Control. For that, it is assumed that the dominant damping effects are located in the contact area close to the adaptive screw and can be modeled by a discrete friction model. Then, the dissipated work $W_{d}$ due to friction during one vibration cycle is maximized to find the control. For the chosen model, the dissipated energy

$$
W_{d}=4\left(u_{\text {rel, } 0}-\frac{F_{C}}{k_{T}}\right) F_{C} \quad \text { with } F_{C}=\mu F_{N}
$$

is maximized to yield the optimal normal force $F_{N}$ as a function to the tangential contact stiffness $k_{T}$, the friction coefficient $\mu$, and the relative sliding oscillation amplitude $u_{\text {rel }, 0}$ :

$$
F_{N}=f\left(u_{\mathrm{rel}, 0}\right)=\frac{k_{T} u_{\mathrm{rel}, 0}}{2 \mu}=k_{T}^{*} u_{\mathrm{rel}, 0} .
$$

Note that similar algebraic expressions could be derived also based on hysteresis loops of more complex friction models involving more parameters. Equation (3) is interpreted as control law to adjust the normal force $F_{N}=F_{N, 1}$ according to Figure 1 to the structural vibration tangential amplitude. The required actual vibration displacement amplitude $u_{\text {rel, } 0}$ can be found under the assumption of monofrequent displacement (with zero mean) by $u_{\text {rel, } 0} \approx(\pi / 2 T) \int_{t-T}^{t}\left|u_{\text {rel }}\right| d \tau$ from 


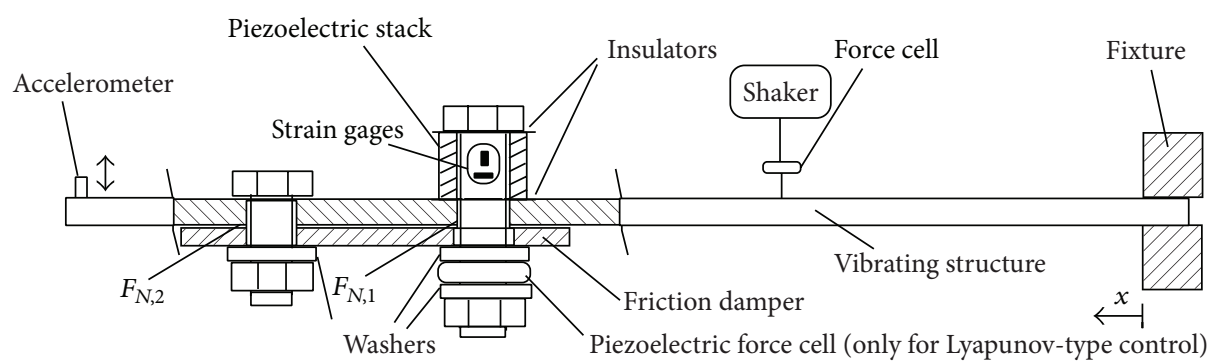

FIGURE 1: Sketch of the benchmark structure (steel, $775 \mathrm{~mm}$ length, $40 \mathrm{~mm}$ width, and $3 \mathrm{~mm}$ thickness) with adaptive friction damper beam (steel, $160 \mathrm{~mm}$ length, $40 \mathrm{~mm}$ width, and $3 \mathrm{~mm}$ thickness).

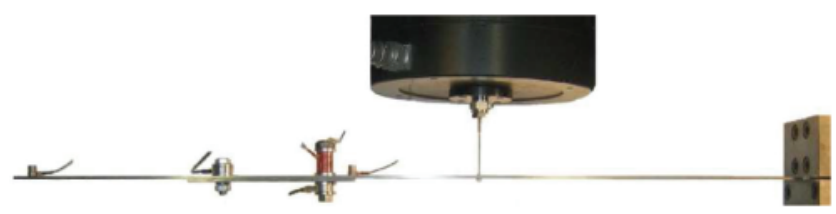

FIGURE 2: Photograph of the experimental setup with structure, damper beam, piezoelectric stack, force cell, accelerometers, and attached shaker stinger (cf. Figure 1).

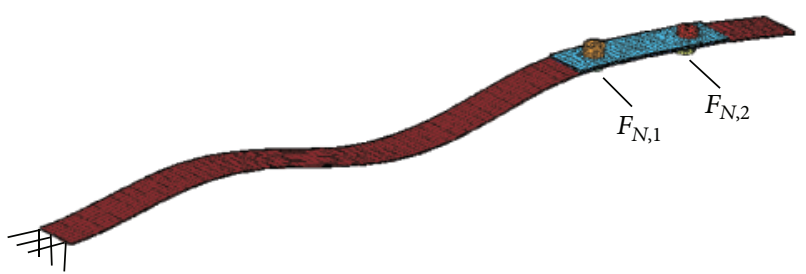

FIGURE 3: Finite-element model $(\approx 30000$ DOFs and 632 nodes in contact) in typical bending deformation with the two screws that impose the external clamping force pairs $F_{N, 1}$ and $F_{N, 2}$.

the estimated signal $u_{\text {rel }}$. The evaluation of the integral would need large memory storage for the required integration time much larger than the largest vibration period of interest; hence it is efficiently approximated by a first-order lag element $\left(P T_{1}\right)$, where the time constant $T_{1}$ prescribes how fast the signal $u_{\text {rel, } 0}$ follows a change in the vibration amplitude:

$$
T_{1} \dot{u}_{\mathrm{rel}, 0}+u_{\mathrm{rel}, 0}=\frac{\pi}{2}\left|u_{\mathrm{rel}}\right| .
$$

3.2. Model-Based Design of Nonlinear Observer. In general, the required tangential displacement $u_{\text {rel }}$ at the adaptive screw cannot be measured which makes the design of a nonlinear observer necessary which estimates it from available measurements such as displacements, strains, or accelerations and a simplified approximate simulation model without having any information about the excitation forces.

This model is derived by rigid connection of the damper beam at one end $\left(F_{N, 2}\right)$ and neglect of the normal contact between damper and base structure; instead appropriate spring elements are introduced in normal direction in some distance around the adaptive screw. The relative motion at the adaptive screw is used for the output definition of $\widehat{u}_{\text {rel }}$. The nonlinear observer is of the form

$$
\begin{aligned}
\dot{\bar{z}}= & A \widehat{z}+B_{T} f_{\text {friction }}\left(\widehat{F}_{T}, \dot{\hat{u}}_{\text {rel }}, F_{N}\right)+B_{\text {exc }} F_{\text {exc }} \\
+ & l\left(y_{\text {meas }}-\widehat{y}_{\text {meas }}\right), \\
& \text { with } \widehat{y}_{\text {meas }}=C \widehat{z}, \quad \widehat{u}_{\text {rel }}=C_{\text {rel }} \widehat{z} .
\end{aligned}
$$

Hereby, the measurement output $y_{\text {meas }}$ denotes the velocity measurement obtained by integration of the acceleration in the experiment. The estimated output $\widehat{u}_{\text {rel }}$ replaces the required variable $u_{\text {rel }}$ by the nonlinear control law (3). A simple dynamic friction model $f_{\text {friction }}(\cdot)$ is used to model the friction $F_{T}$ at the adaptive screw, namely, a regularized Coulomb friction model:

$$
F_{T}=f_{\text {friction }}\left(\dot{\hat{\vec{u}}}_{\text {rel }}, F_{N}\right)=\mu F_{N} \frac{2}{\pi} \arctan \left(\alpha \dot{\hat{\vec{u}}}_{\text {rel }}\right),
$$

which depends on the relative velocity $\dot{\hat{u}}_{\text {rel }}$ with the regularization parameter $\alpha$ determined by simulation studies. Theoretically, the use of more complex dynamic friction models would be of interest; the hard real-time limitations imposed by the fixed-step time-integration in the experiment and the considered high frequencies forbid their application for the investigated problem. The obtained estimation accuracy has been verified in simulations that are also used to optimize the observer. The linear system parts in (5) are obtained from a simplified, linear FE model after modal truncation plus a static correction step. With the required output variables, mass and stiffness matrices $M, K$ and load vector $F_{\text {exc }}$, this simplified model reads with nonlinear inner force vector $F_{I}$ consisting of normal contact and friction forces

$$
\begin{aligned}
M \ddot{x}+K x+F_{I}= & \beta_{\mathrm{exc}} F_{\mathrm{exc}}, \quad y_{\text {meas }}=\gamma_{\text {meas }} x, \\
& u_{\text {rel }}=\gamma_{\text {rel }} x .
\end{aligned}
$$

Solving the associated eigenvalue problem $\left(K-\omega^{2} M\right) \phi=0$ yields the eigenfrequencies $\omega_{k}$ and eigenvectors $\phi_{k}\left(k \in \mathbb{N}^{+}\right)$ which allows a modal truncation to the first $N$ important bending modes by the transformation $x=T x^{*}$ with $T=$ $\left[\phi_{1}, \phi_{2}, \ldots, \phi_{N}\right]$. For a real-time application, an observer of low order is aspired. Additional information to identify important bending modes which are observable can be obtained by modal Gramians; see [15, 17]. This transforms 


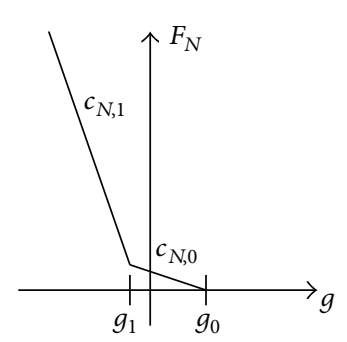

(a)

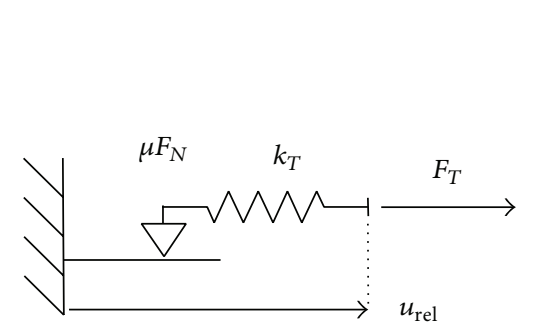

(b)

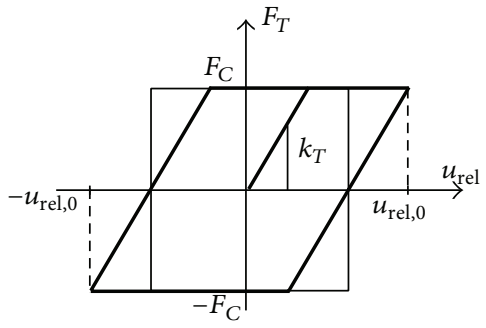

(c)

Figure 4: (a) Normal force stiffness relation. (b) 1D Jenkins friction model. (c) Jenkins model hysteresis loop (thick) in comparison to the Coulomb friction hysteresis (thin). Note, that for increasing tangential stiffness, the hysteresis approximates the Coulomb model hysteresis.
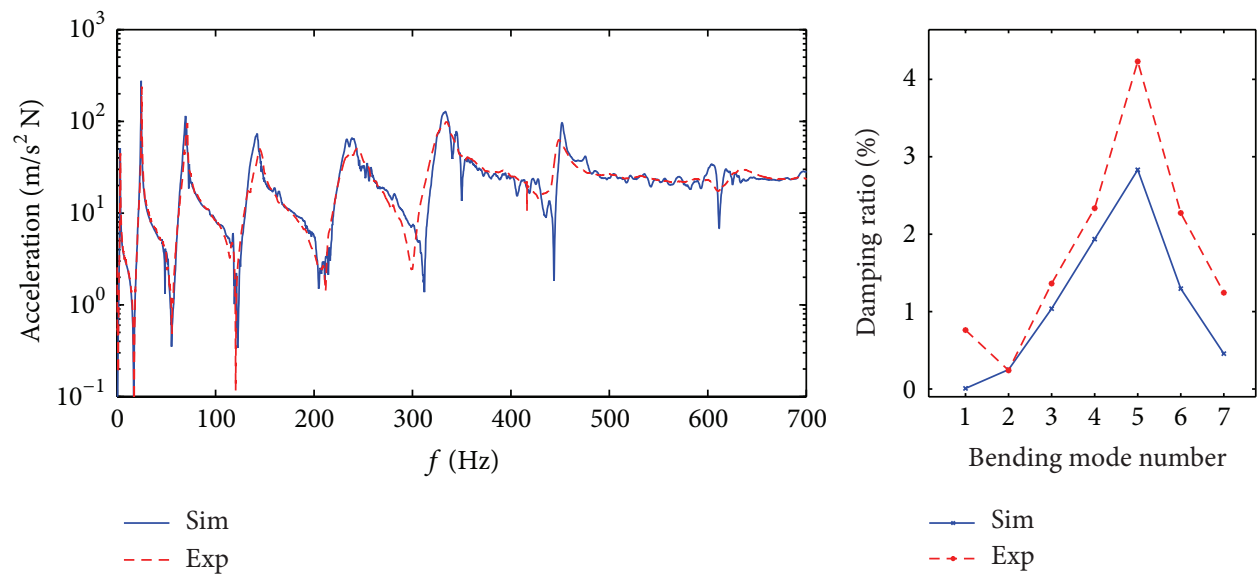

FIGURE 5: Measured and simulated FRFs for impulse excitation and $F_{N, 1}=100 \mathrm{~N}, F_{N, 2}=6000 \mathrm{~N}$ (excitation and measurement close to tip on the mid-axis) and determined modal damping ratios.

the system matrices, $I=T^{T} M T$ and $\Omega=T^{T} K T=\operatorname{diag}\left\{\omega_{1}^{2}\right.$, $\left.\omega_{2}^{2}, \ldots, \omega_{N}^{2}\right\}$, as well as the other matrices in (5). With the state vector $z^{T}=\left[\dot{x}^{* T}, x^{* T}\right]$ and some damping matrix $\Delta$, these linear system parts read

$$
\begin{gathered}
A=\left[\begin{array}{cc}
-\Delta & -\Omega \\
I & 0
\end{array}\right], \quad B_{T}=\left[\begin{array}{c}
-\Theta^{T} \gamma_{\text {rel }}^{T} \\
0
\end{array}\right], \\
B_{\text {exc }}=\left[\begin{array}{c}
\Theta^{T} \beta_{\text {exc }} \\
0
\end{array}\right], \quad C_{\text {rel }}=\left[0, \gamma_{\text {rel }} \Theta\right], \\
C_{\text {meas }}=\left[0, \gamma_{\text {meas }} \Theta\right] .
\end{gathered}
$$

After linearization around $u_{\text {rel }}=0, F_{N}=0$, the observer gains $l$ in (5) are determined by a Kalman design procedure from the solution of the associated Riccati equation for appropriate state and measurement noise variance matrices. The obtained control loop is shown in Figure 6.

3.3. Lyapunov-Type Control. For the second proposed control law, Lyapunov's direct method is applied by choosing the mechanical system energy as Lyapunov function $V(x)$ and under the assumption of a discrete friction model with controlled normal force [13]. It is imposed that its time derivative $\dot{V}(x)$ must be seminegative, which is directly related to the differential form of the passivity condition.
Its absolute value is furthermore maximized for optimality in the Lyapunov sense. Recalling the dynamics of a 1-DOF system

$$
m \ddot{u}_{\text {rel }}+k u_{\text {rel }}+F_{T}=0 \text {, }
$$

the passivity condition in terms of the friction force $F_{T}$ and the relative velocity $\dot{u}_{\text {rel }}$

$$
F_{T} \dot{u}_{\text {rel }} \geq 0 \quad \forall t
$$

ensures a power flow outwards of the controlled structure. To enforce (10), a control law based on a Jenkins friction model must depend on the actual friction force $F_{T}$. However, in practice, for structures as the investigated one, it is almost impossible to measure or estimate this force because of the distributed friction interface and the high stiffnesses in combination with the hard real-time constraints. Hence, the Coulomb friction model according to $F_{T}=\mu F_{N} \operatorname{sign}\left(\dot{u}_{\text {rel }}\right)$ is assumed as a good approximation instead. For this, it can be shown that a velocity-dependent bang-bang controller, which switches continuously between the two possible states, is optimal in the Lyapunov sense [13]; $F_{N}=0$ for $\left|\dot{u}_{\text {rel }}\right|=$ 0 and $F_{N}=F_{N, \text { max }}$ for $\left|\dot{u}_{\text {rel }}\right| \geq 0$. This approach is appropriate in view of the high values for the tangential stiffness $k_{T}$ found in the model updating before. Additional regularization with a boundary layer $\varepsilon$ to avoid chattering 


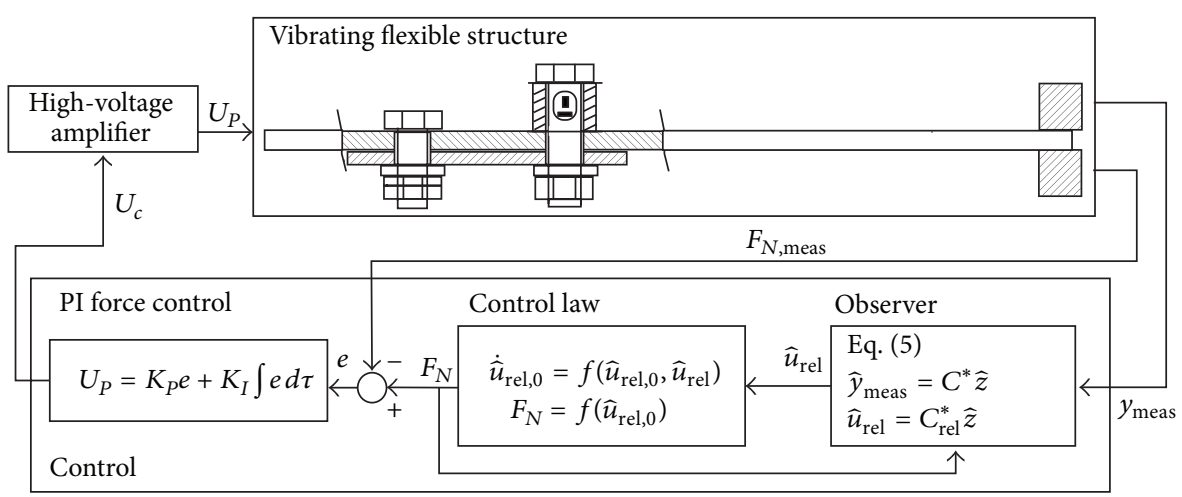

FIGURE 6: Closed control loop with hysteresis-optimal control law.

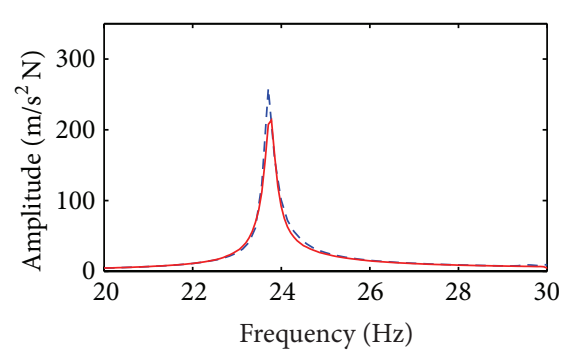

(a) Mode 2, $F_{\text {exc }, 0}=0.4 \mathrm{~N}$

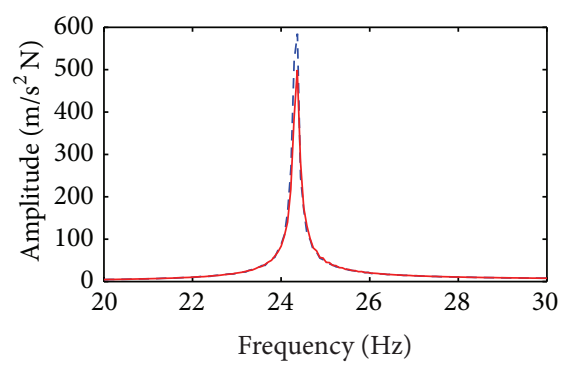

(d) Mode 2, $F_{\text {exc }, 0}=0.4 \mathrm{~N}$

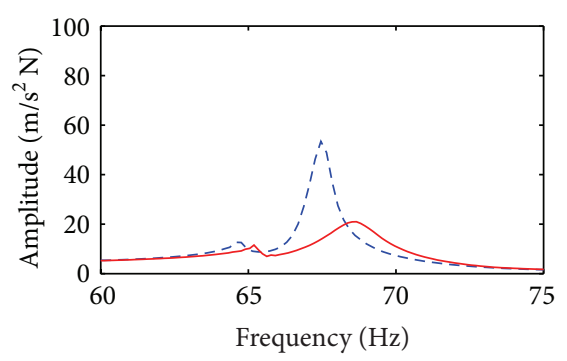

(b) Mode 3, $F_{\text {exc }, 0}=3 \mathrm{~N}$

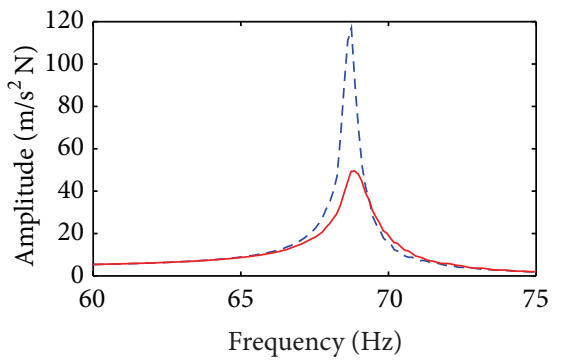

(e) Mode $3, F_{\text {exc }, 0}=3 \mathrm{~N}$

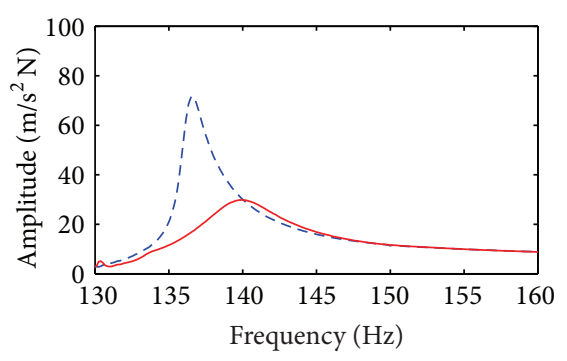

(c) Mode $4, F_{\text {exc }, 0}=3 \mathrm{~N}$

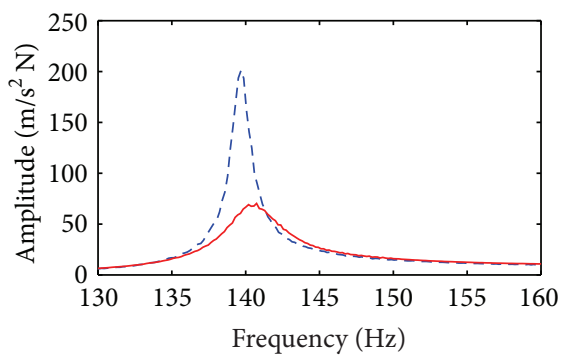

(f) Mode $4, F_{\text {exc }, 0}=3 \mathrm{~N}$

FIGURE 7: Hysteresis-optimal control: measured (top) and simulated (bottom) accelerance FRFs for controlled sine-sweep excitation with (solid) and without (dashed) control $\left(k_{T}^{*}=2.5 \cdot 10^{7} \mathrm{~N} / \mathrm{m}\right)$.

effects and introduction of the minimal normal force yields the suboptimal law:

$$
F_{N}= \begin{cases}\max \left\{\frac{\left|\dot{u}_{\text {rel }}\right|}{\varepsilon} F_{N, \text { max }}, F_{N, \text { min }}\right\} & \text { for }\left|\dot{u}_{\text {rel }}\right|<\varepsilon \\ F_{N, \text { max }} & \text { for }\left|\dot{u}_{\text {rel }}\right| \geq \varepsilon .\end{cases}
$$

Again, the required tangential relative motion must be estimated by the previously introduced observer which now estimated the relative velocity $\dot{u}_{\text {rel }}$ instead of the displacement $u_{\text {rel }}$. Note that the minimal and maximal normal forces $F_{N \text {,min }}$ and $F_{N, \max }$ are determined by the mechanical properties of the adaptive screw.

\section{Experiments and Simulation Results}

The proposed controls are investigated for the benchmark structure with a damper beam at $x_{d}=0.545 \mathrm{~m}$ away from the fixture. For this position, significant relative displacement between structure and damper is expected for the bending modes 3, 4 and higher. For mode 2, much less relative motion is expected which explains the small damping values obtained in the experiment and simulations, for example, for the passive results in Figure 5. The first mode is not considered because it cannot be excited by the available shaker. For evaluation, accelerated FRFs from the excitation force (at $x=$ $0.325 \mathrm{~m}$ ) to the measured acceleration (at $x=0.45 \mathrm{~m}$ ) are compared.

4.1. Controller Implementation. A dSpace system running at $21 \mathrm{kHz}$ sampling frequency is used for the real-time implementation. The observer is designed based on 7 normal modes and uses the out-of-plane tip acceleration at $x=$ $0.765 \mathrm{~m}$ as measurement variable $y_{\text {meas }}$. In the experiment, the prescribed force $F_{N}$ for the adaptive screw from (3) or 


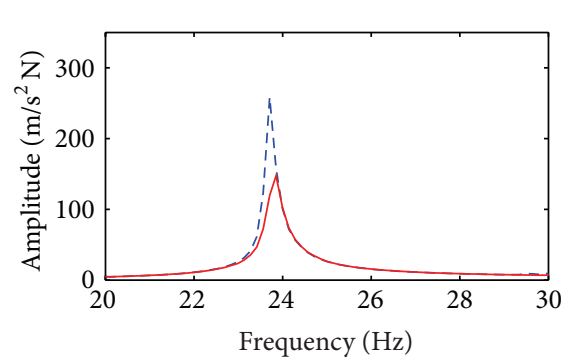

(a) Mode 2, $F_{\text {exc }, 0}=0.4 \mathrm{~N}$

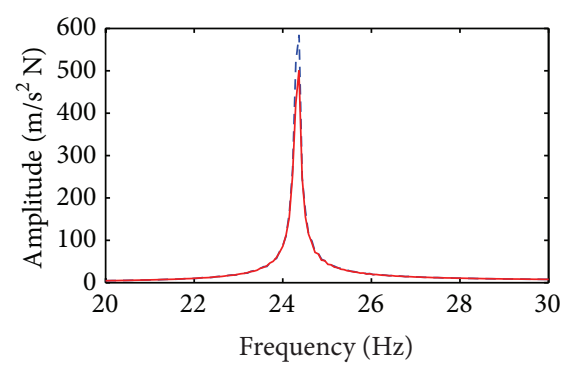

(d) Mode 2, $F_{\text {exc }, 0}=0.4 \mathrm{~N}$

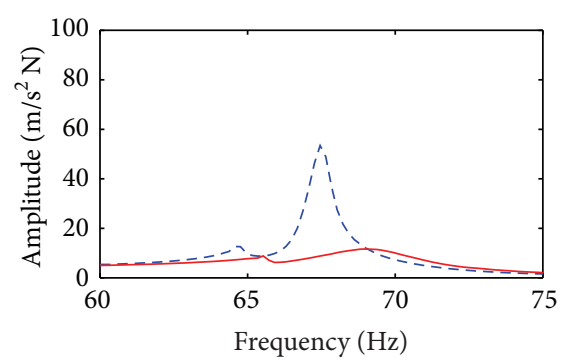

(b) Mode $3, F_{\text {exc }, 0}=3 \mathrm{~N}$

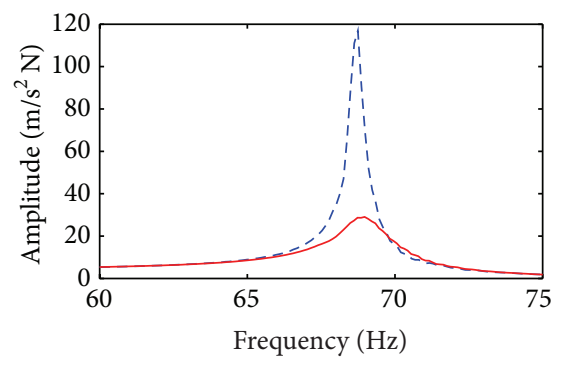

(e) Mode 3, $F_{\text {exc }, 0}=3 \mathrm{~N}$

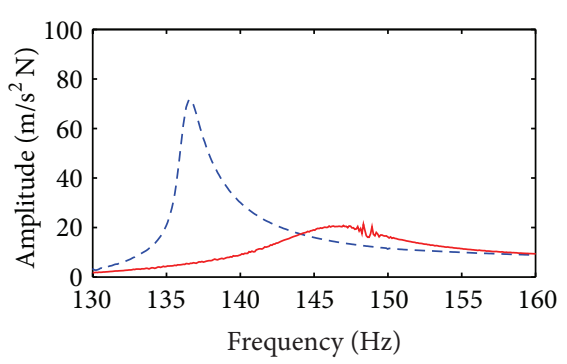

(c) Mode $4, F_{\text {exc }, 0}=3 \mathrm{~N}$

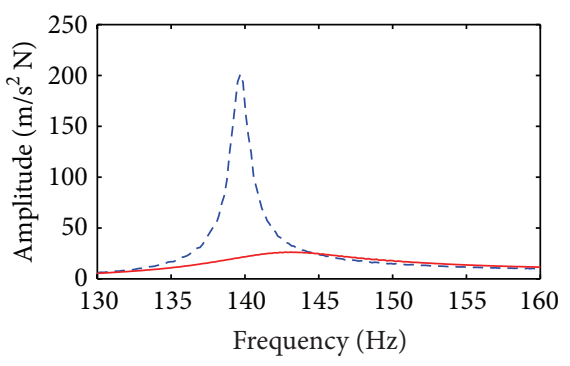

(f) Mode $4, F_{\text {exc }, 0}=3 \mathrm{~N}$

FIGURE 8: Lyapunov-type control: measured (top) and simulated (bottom) accelerance FRFs for controlled sine-sweep excitation with (solid) and without (dashed) control $(\varepsilon=1 / 200 \mathrm{~m} / \mathrm{s})$.

(11) must be tracked by an underlying force feedback control loop to compensate nonlinearities, large-signal piezoelectric effects, and creep effects in the screw threads as well as decrease the inertia forces of the actuator and sensor masses due to the structural vibrations. For that, a tracking controller is employed which is combined from a feedforward term derived from the static actuator voltage-force relationship and a PID feedback control of the measured force. The obtained actuator signal $U_{P}$ is filtered by a $30 \mathrm{kHz}$ low-pass filter to decrease the digitization noise before it is amplified for the piezoelectric stack actuator. To maximize the stiffness of the clamping of the piezoelectric actuators determining the achievable stroke [2], strain gages directly embedded in the bolt shaft (see Figure 6) measure the actual force instead of strain-gage based ring force cells that would significantly weaken the configuration. The applicable actuator stroke lies between $F_{N, \text { min }}=40 \mathrm{~N}$ and $F_{N, \text { max }} \approx 600 \mathrm{~N}$.

For the Lyapunov-type control, a piezoelectric force cell of high sensitivity and bandwidth is added (cf. Figure 2) because its high actuation dynamics requires a very high control bandwidth which is difficult to realize with strain-gage based force measurements due to the found signal-to-noise ratio and delays originating from the necessary amplifiers.

4.2. Shaker Test Setup with Excitation Force Control. For nonlinear mechanical structures, comparing FRFs requires special care because the obtained FRFs are nonlinear. More specifically, their resonance frequencies, peak amplitudes, and peak forms depend on the excitation signal as well as amplitudes. Consequently, the amplitude is controlled during sine sweep measurements to make the excitation independent of the structural impedance for consistent comparisons.
Very low sweep velocities $(0.1 \mathrm{~Hz} / \mathrm{s})$ are employed to obtain steady-state conditions which approximate step-sine testing and to avoid interaction between the interesting effects of the semiactive structural control and the shaker control. Due to the very small relative displacements outside resonances, the control is only effective close to resonances which allows restricting the evaluation around the resonance frequencies to save simulation and measurement time.

In Figures 7 and 8, FRFs with and without semiactive control are shown for some typical excitation amplitudes for the two control concepts. Similar results are obtained for other amplitudes. It can always be seen that the control strongly reduces the resonance amplitudes of multiple modes. However, for the second mode the obtained damping is quite small in the active as well as the passive case. This is due to the slight curvature of the beam at the lower modes which results in a small relative motion in the interface. Additionally, this fact implies that the obtained efficiency of the damper is dependent on its position.

In the passive case, the minimal possible force $F_{N}=$ $F_{N \text {,min }}$ is applied to the adaptive screw which still introduces significant structural damping compared to the case without attached damper. Experiments and simulation additionally prove that the semiactive control never decreases the passive damping effect at lower excitation amplitudes. Generally, the agreement obtained between experiment and simulations is rated very good in view of the well-known difficulties encountered in the prediction of nonlinear damping of structures, especially for distributed friction with inhomogeneous normal contact pressure distribution. Furthermore, some imperfections of the excitation control cannot be avoided as well as some changes in the contact parameters. The Lyapunov-type control achieves higher vibration reduction 
than the hysteresis-optimal one in both experiments and simulations, especially at smaller vibration amplitudes. It is also suited to suppress broadband vibrations and relatively robust to errors in the estimation, but, as a drawback, its high dynamical actuation requires more power. Advantageously, the hysteresis-optimal control could be implemented with low dynamical actuators, for example, of different actuation principles.

\section{Conclusion}

Multimodal, semiactive vibration controllers that adapt the normal force applied to friction damper beams by piezoelectric stack actuators are investigated for a generic benchmark structure in experiments and simulations. They are shown to efficiently damp structural resonances for different excitation amplitudes and vibration modes. Which of the investigated controller concepts suits best for a certain application depends mainly on the actuator principle, the power considerations, and whether the excitation being rather broadband or narrowband. Based on the results of the beam experiment, the proposed friction damper is used to reduce the vibrations of machine tools [18].

\section{Conflict of Interests}

The authors declare that there is no conflict of interests regarding the publication of this paper.

\section{Acknowledgment}

The support of the DFG (German Research Foundation) with Project SPP 1156 is gratefully acknowledged.

\section{References}

[1] M. J. Balas, "Feedback control of flexible systems," IEEE Transactions on Automatic Control, vol. AC-23, no. 4, pp. 673-679, 1978.

[2] C. Fuller, S. Elliott, and P. Nelson, Active Control of Vibration, Academic Press, New York, NY, USA, 1996.

[3] P. Dupont, P. Kasturi, and A. Stokes, "Semi-active control of friction dampers," Journal of Sound and Vibration, vol. 202, no. 2, pp. 203-218, 1997.

[4] L. M. Jansen and S. J. Dyke, "Semiactive control strategies for MR dampers: a comparative study," Journal of Engineering Mechanics, vol. 126, no. 8, pp. 795-803, 2000.

[5] O. E. Ozbulut, M. Bitaraf, and S. Hurlebaus, "Adaptive control of base-isolated structures against near-field earthquakes using variable friction dampers," Engineering Structures, vol. 33, no. 12, pp. 3143-3154, 2011.

[6] S. Nagarajaiah, S. Narasimhan, A. Agrawal, and P. Tan, "Semiactive Lyapunov controller for phase II seismic isolated highway bridge benchmark," in Proceedings of the Structures Congress, pp. 1-10, 2006.

[7] F. Nitzsche, D. G. Zimcik, V. K. Wickramasinghe, and C. Yong, "Control laws for an active tunable vibration absorber designed for rotor blade damping augmentation," Aeronautical Journal, vol. 108, no. 1079, pp. 35-42, 2004.
[8] W. N. Patten, C. Mo, J. Kuehn, and J. Lee, "A primer on design of semiactive vibration absorbers (SAVA)," Journal of Engineering Mechanics, vol. 124, no. 1, pp. 61-67, 1998.

[9] H. Li, J. Li, and G. Song, "Improved suboptimal Bang-Bang control of aseismic buildings with variable friction dampers," Acta Mechanica Sinica, vol. 23, no. 1, pp. 101-109, 2007.

[10] M. D. Symans and M. C. Constantinou, "Seismic testing of a building structure with a semi-active fluid damper control system," Earthquake Engineering and Structural Dynamics, vol. 26, no. 7, pp. 759-777, 1997.

[11] M. Unsal, C. Niezrecki, and C. Crane III, A New Semi-Active Piezoelectric-Based Friction Damper, Department of Mechanical Engineering, University of Florida, 2007.

[12] J. S. Lane, A. A. Ferri, and B. S. Heck, "Vibration control using semi-active friction damping," in Proceedings of the Winter Annual Meeting of the American Society of Mechanical Engineers (ASME '92), vol. 49, pp. 165-171, November 1992.

[13] R. Nitsche and L. Gaul, "Lyapunov design of damping controllers," Archive of Applied Mechanics, vol. 72, no. 11-12, pp. 865874, 2003.

[14] J. Becker and L. Gaul, "CMS methods for efficient damping prediction for structures with friction," in Proceedings of the 26th Conference and Exposition on Structural Dynamics (IMACXXVI '08), Orlando, Fla, USA, February 2008.

[15] J. Becker, Semi-active control of friction dampers and feedforward tracking control design for structural vibration reduction [Ph.D. thesis], University of Stuttgart, Der Andere, 2009.

[16] L. Gaul and R. Nitsche, "The role of friction in mechanical joints," Applied Mechanics Reviews, vol. 54, no. 2, pp. 93-105, 2001.

[17] W. K. Gawronski, Dynamics and Control of Structures, Mechanical Engineering Series, Springer, New York, NY, USA, 1998.

[18] J. Hesselbach, Adaptronik für Werkzeugmaschinen: Forschung in Deutschland, Shaker, 2011. 

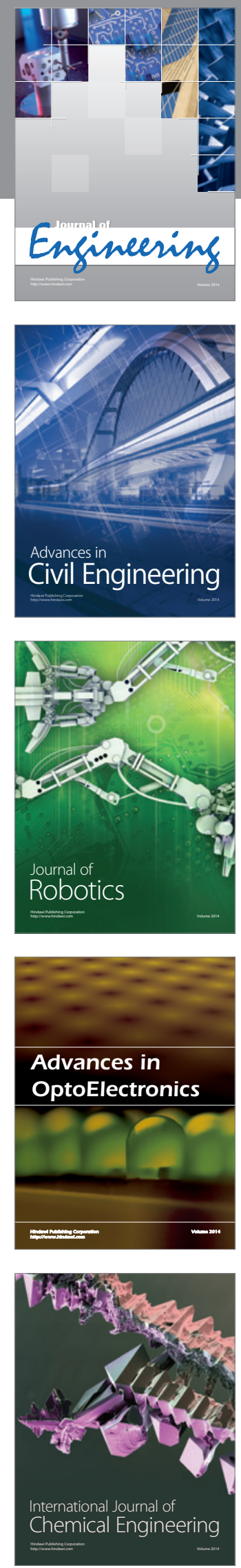

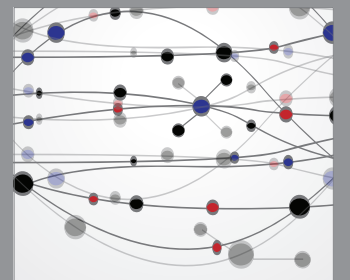

The Scientific World Journal
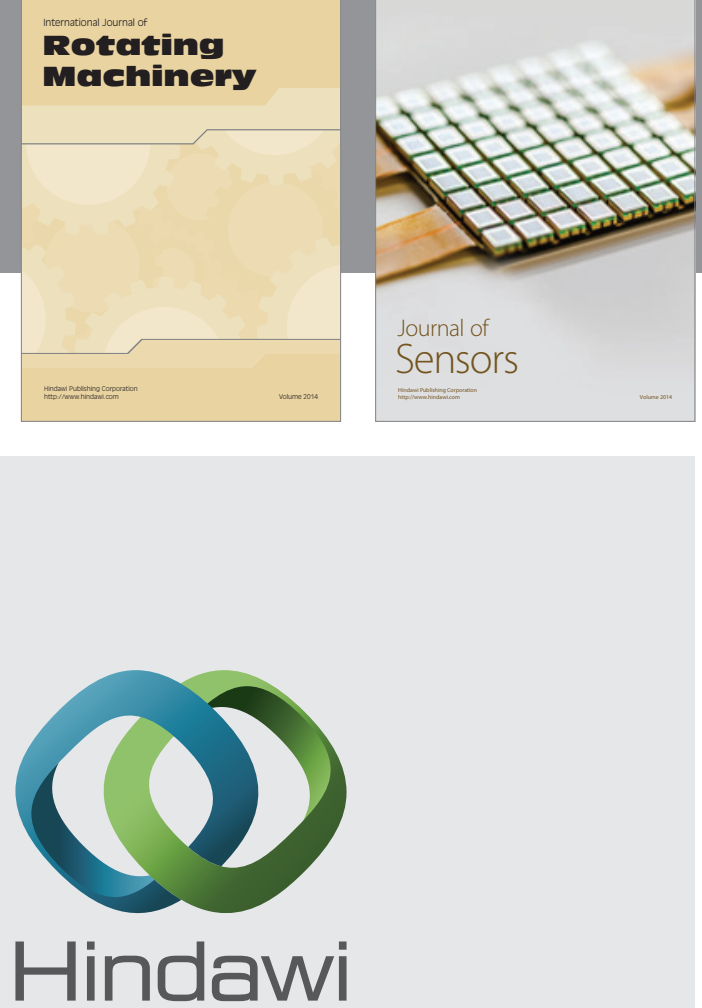

Submit your manuscripts at http://www.hindawi.com
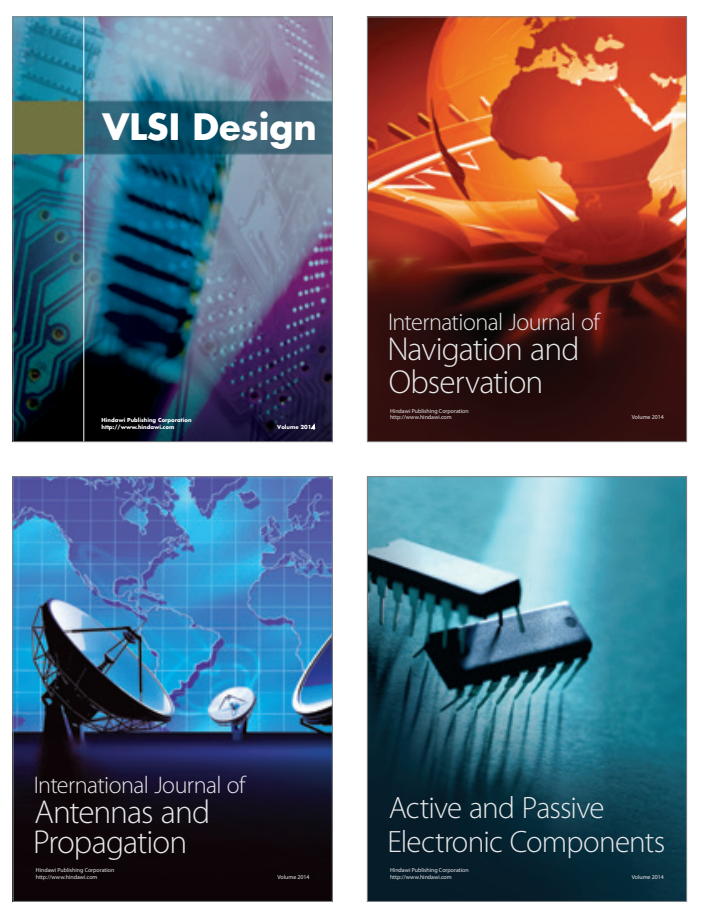
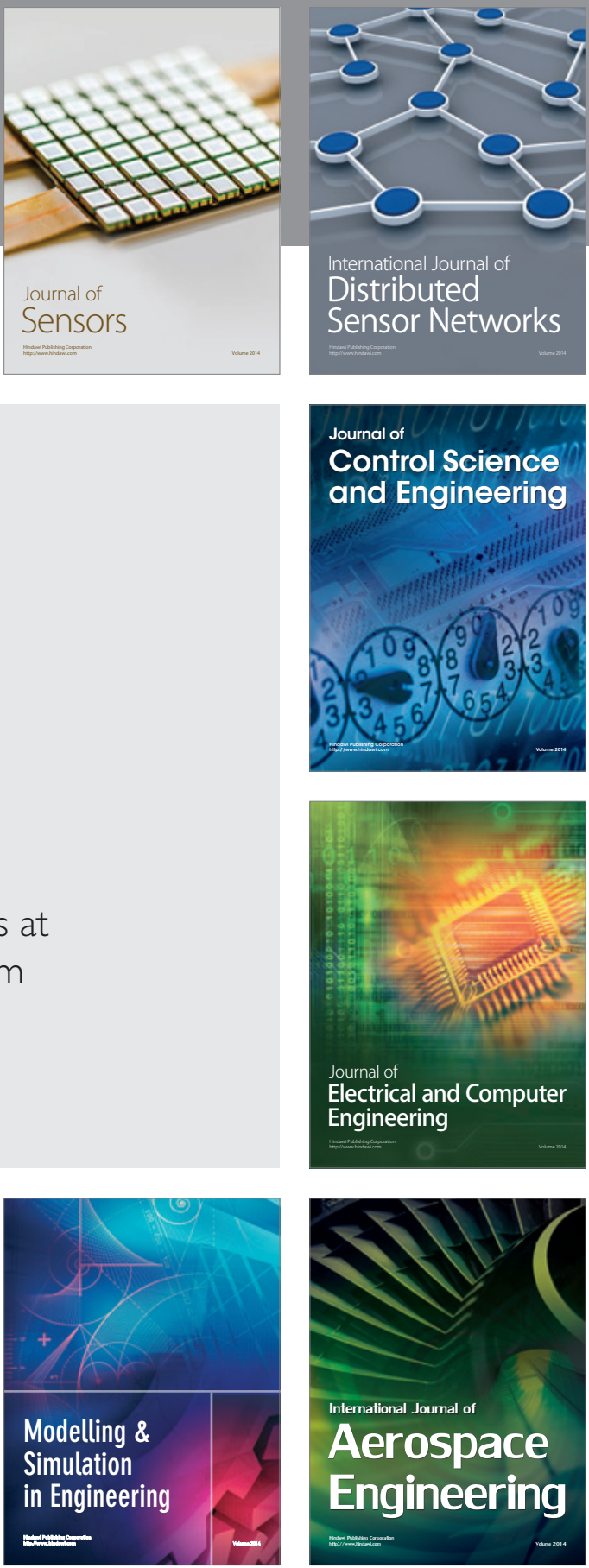

Journal of

Control Science

and Engineering
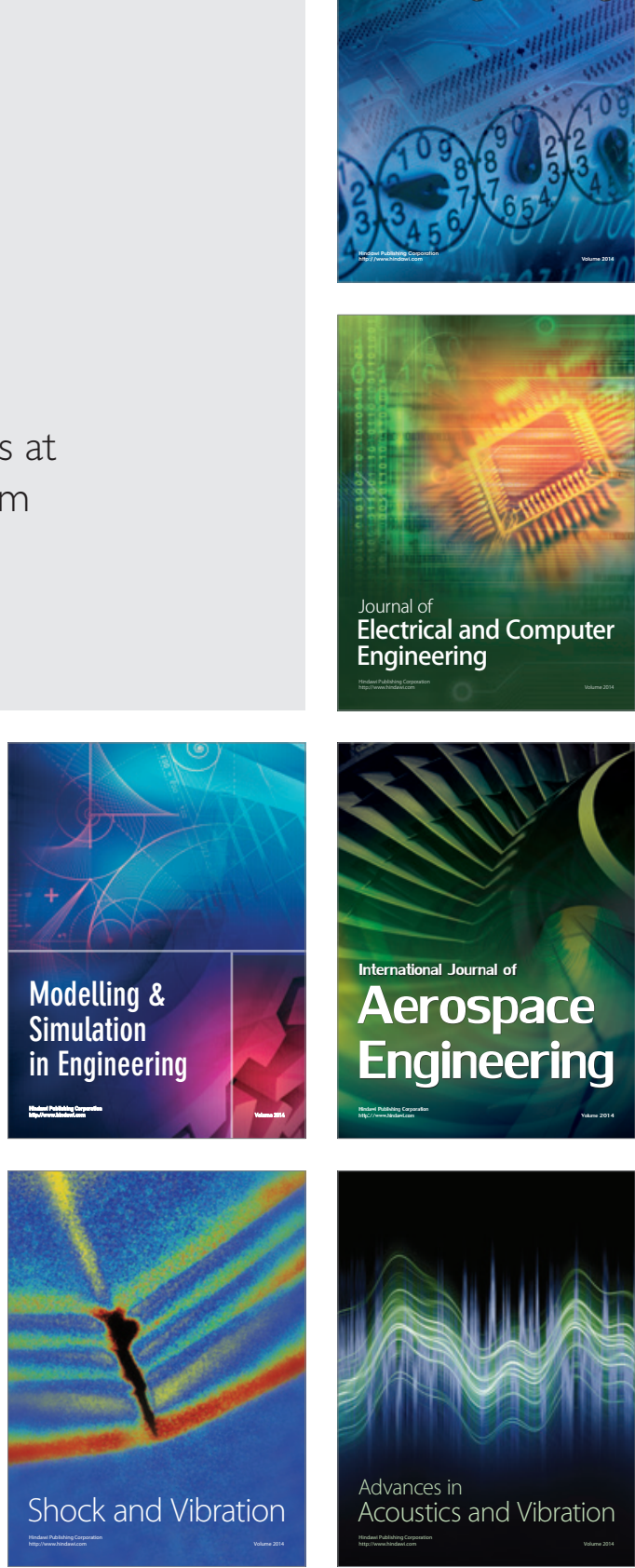